\title{
Novel protocol for synthesis of 1,4-diiminocurcumin stabilized silver nanoparticles and application as heterogenous recyclable catalyst and antibacterial agent
}

\author{
NISHI GANDHA GOGOI and JYOTIREKHA G HANDIQUE* (B) \\ Department of Chemistry, Dibrugarh University, Dibrugarh 786 004, Assam, India \\ E-mail: jghandique@rediffmail.com; ngogoi28@gmail.com
}

MS received 16 March 2019; revised 28 May 2019; accepted 28 May 2019

\begin{abstract}
Curcumin [(1E, 6E)-1,7-bis(4-hydroxy-3-methoxy-phenyl)-1,6-heptadiene-3,5-dione] is a low molecular weight yellow-orange polyphenolic pigment extracted from the powdered rhizome of Curcuma longa. Curcumin has wide medicinal applications as an antioxidant, anti-inflammatory, cancer chemopreventive, and potentially chemotherapeutic agents as well as stabilizer/reducing agent in silver nanoparticles (AgNPs) synthesis. However, the low solubility of curcumin in aqueous solutions limits its applications and also, many of AgNP synthetic processes lack a greener synthetic route. In the present work, a Schiff base of curcumin is synthesized condensing curcumin and 1,4-diaminobutane in 2:1 ratio. The resulting product shows improvement in solubility in water and favours the synthesis of AgNPs in aqueous medium at room temperature, acting as a self-reducing/stabilizing agent. This proposed synthetic route is simple, feasible and green. The size and morphology of AgNPs are analyzed by TEM, SEM, EDS and XRD techniques. The recyclable AgNPs as a heterogeneous catalyst in the reduction of nitroaromatics to amino compounds is environmentally benign and can be re-used up to $5^{\text {th }}$ cycle without considerable loss of its catalytic activity. Moreover, both Cur-1,4 and AgNPs show bactericidal properties against bacterial strains (Bacillus subtilis, Staphylococcus aureus, Escherichia coli and Pseudomonas aeruginosa) which find medicinal importance in future.
\end{abstract}

Keywords. Curcumin; schiff base of curcumin; silver nanoparticles; green synthesis; reduction of nitroaromatics; antibacterial property.

\section{Introduction}

Natural polyphenolic compound, curcumin, a yelloworange pigment, extracted from the rhizome of Curcuma longa (turmeric) $)^{1,2}$ is extensively used as herbal supplement and food flavouring/colouring agent. ${ }^{3}$ It shows potent pharmacological properties in pre-clinical processes as antimicrobial, antiviral, antioxidant, anti-inflammatory, anticancer, anti-HIV, anti-Alzheimer's and anti-depressant. ${ }^{4-6}$ Curcumin has proven its ability in chemotherapeutic and cancer and neurological disease prevention. ${ }^{7-10}$ On the basis of structureactivity-relationship (SAR) studies on curcumin molecule, it is known that the phenolic hydroxyl $(-\mathrm{OH})$ groups of curcumin is responsible for showing its antioxidant property. ${ }^{11}$ This qualifies curcumin to inhibit oxidative stress in red blood cell membranes, provide protection against degenerative cells, and induce enzyme detoxification by scavenging free radicals. $^{12}$ But poor biological availability, chemical instability, and low aqueous solubility limit the wide medicinal, clinical and pharmacological applications of curcumin. ${ }^{13-15}$ Various techniques have been developed to tackle with aqueous insolubility of curcumin by encapsulating curcumin in form of polymeric micelles, solid support nanoparticles, polymeric nanoparticles, biodegradable microspheres, phospholipids, cyclodextrin, hydrogel, and liposomes. ${ }^{16-20}$ Recently, various synthetic derivatives of curcumin, such as Schiff bases of curcumin ${ }^{21,22}$ were synthesized by modification of the central $\beta$-diketone moiety, thereby enhancing the chemical/physical stability and

*For correspondence

Electronic supplementary material: The online version of this article (https://doi.org/10.1007/s12039-019-1654-1) contains supplementary material, which is available to authorized users. 
aqueous solubility compared to curcumin maintaining its medicinal value. ${ }^{13,23,24}$

In recent times, greener alternatives for the synthesis of silver nanoparticles (AgNPs) are given utmost importance considering the associated environmental issues from the conventional synthetic methodologies. AgNPs are known to have medicinal values from ancient times. ${ }^{25}$ The antibacterial properties of AgNPs are conventionally used in medicines to reduce bacterial infections in burn and wounds, and arthroplasty, ${ }^{26}$ to arrest bacterial growth on human skin, in medical equipment and materials, and in water treatment and textile industries. ${ }^{27}$ But AgNPs often tend to agglomerate and form larger-sized particles without a support/ stabilizer. In this way, their surface energy is decreased followed by their other beneficial usability. AgNPs unattached to a support/stabilizer is difficult to recover and reuse when used as a catalyst. Therefore various stabilizers such as artificial polymers [polyurethanes (PU), polyvinylchloride (PVC), polyacrylamides and polyethylene glycols (PEGs)], ${ }^{28-32}$ ionic surfactants [Sodium dodecyl sulphate (SDS), and cetyltrimethylammonium chloride or bromide (CTAC or CTAB)], ${ }^{33}$ and non-ionic surfactants (Brij, Tween, and Trinton $\mathrm{X}-100)^{34}$ in AgNPs preparation (interaction with nanoparticles is based on steric and electrostatic repulsion of polymers) $)^{35}$ are commonly used in showing persistence of the antibacterial property of AgNPs along with its optical, electronic and catalytic properties. ${ }^{36-38}$ Many conventional methods for the preparation of AgNPs incorporate dangerous chemicals (as borohydrides or hydrazines as reducing agents) and consumption of a large amount of electrical/mechanical energy and formation of hazardous byproducts. Thus, new and greener approaches using environment-friendly reducing agents, non-toxic stabilizers and green solvents for the generation of AgNPs are encouraged. ${ }^{39}$ Approaches towards the synthesis of nanoparticles using natural products and in an aqueous medium, without the need of external reducing agent are intensively pursued. Native curcumin and its nanocomposite are found to act as stabilizers in preparation of AgNPs. ${ }^{14,36,40,41}$

In this work, synthesis of a novel Schiff base of curcumin (Cur-1,4) with 1,4-diaminobutane (having a biological origin, putrescine) is reported. The synthesized Schiff base, Cur-1,4, shows enhancement in aqueous solubility compared to curcumin. The formation Cur-1,4 was confirmed by Fourier transform infrared (FTIR) and ${ }^{1} \mathrm{H}$ and ${ }^{13} \mathrm{C}$ NMR spectroscopy. Cur-1,4 acts as a self-stabilizing as well as a reducing agent in green synthesis of AgNPs in water and at room temperature. The elemental analysis in stabilized AgNPs was determined by energy-dispersive X-ray spectroscopy (EDS) pattern. The morphology, size, shape and size distribution of the stabilized AgNPs were analyzed by $\mathrm{X}$-ray diffraction (XRD), transmission electron microscopy (TEM) and scanning electron microscopy (SEM). We have studied the catalytic activity of stabilized AgNPs in the reduction of nitroaromatic compounds at room temperature and in an aqueous medium. The degradation of nitroaromatic compounds was monitored by UV-visible spectrophotometric technique. The antibacterial efficacy of Cur-1,4 and stabilized AgNPs were tested by in vitro inhibition assessment of two gram-positive bacteria (Bacillus subtilis and Staphylococcus aureus) and two Gram-negative (Escherichia coli and Pseudomonas aeruginosa) bacterial strains.

\section{Experimental}

\subsection{Materials and methods}

Curcumin $(>98 \%)$ and 1,4-diaminobutane $(>96 \%)$ were obtained from Sigma-Aldrich Chemicals Inc. (St. Louis, MO, USA). Silver nitrate $(>98 \%)$ and 4- nitrophenol ( $>98 \%$ ) were obtained from E. Merck India Pvt. Ltd. and acetic acid $\left(\mathrm{CH}_{3} \mathrm{COOH}\right)$ and sodium borohydride $\left(\mathrm{NaBH}_{4}\right)$ were obtained from Spectrochem, India. The other nitroaromatic substrates, viz., 1-chloro-3-nitrobenzene, 1bromo-4-nitrobenzene, 1-iodo-4-nitrobenzene, 3-nitrobenzaldehyde, 4-nitrobenzyl alcohol, 2-chloro-3-nitropyridine, 4-nitroaniline, 4-nitrothioanisole were purchased from TCI Chemicals (India) Pvt. Ltd. The solvents used were of HPLC grade. ${ }^{1} \mathrm{H}$ and ${ }^{13} \mathrm{C}$ NMR spectra were recorded on a Bruker Avance II $400 \mathrm{MHz}$ spectrometer in Methanol- $\mathrm{d}_{4}$. Chemical shifts are reported in ppm relative to TMS for NMR spectra. X-ray diffraction pattern (XRD) of stabilized nanosilver were recorded on AXS D8 Advance Diffractometer with $\mathrm{Cu}-\mathrm{K}$ (1.541 $\AA$ radiation). FTIR spectra were recorded in $\mathrm{KBr}$ pallets on a Shimadzu IRPrestige-21 FTIR spectrophotometer $\left(200-4000 \mathrm{~cm}^{-1}\right)$. Transmission electron Microscopy (TEM) analysis of stabilized AgNP were performed on JEM-100 CX II instrument equipped with a high accelerating voltage of 20-100 $\mathrm{KV}$ in $20 \mathrm{KV}$ steps and a high-resolution $\mathrm{CCD}$ camera from $3 \AA$ to $1.4 \AA$. Scanning Electron Microscopy (SEM) and SEM-EDX of the catalysts was recorded on JEOL JSM 7100F. The catalytic activity of Cur-1,4 stabilized AgNPs in the reduction of nitroaromatics was monitored by a UV-Visible Spectrophotometer HITACHI U-3900H. The reusability of AgNPs as catalyst was determined by the $\mathrm{Ag}$ loading in the catalyst and determined using inductively coupled plasma-atomic emission spectroscopy (ICP-AES) analysis on a Thermo Electron IRIS INTERPID II XSP DUO. The mass spectra were recorded in Agilent 7820A GC system. 


\subsection{Synthesis of Cur-1,4}

$2 \mathrm{mmol}$ of curcumin is dissolved in ethanol and then mixed with $1 \mathrm{mmol}$ of 1,4-diaminobutane. A catalytic amount 1-2 drops of acetic acid was added and the reaction mixture was refluxed for $6 \mathrm{~h}$. The solid product obtained was subsequently filtered, fully washed with ethanol, purified and dried at room temperature. The product was re-crystallised from ethanol to get brick red crystals (Cur-1,4) shown in Figure S1 (Supplementary Information).

2.2a Spectroscopic data for characterization of Cur-1,4: Product is isolated as dark red colored crystals; yield $=85 \%$. FTIR $v_{\max }\left(\mathrm{cm}^{-1}\right): \mathrm{O}-\mathrm{H}, 3948 ; \mathrm{C}-\mathrm{H}$ (aromatic) $2990 ; \mathrm{C}=\mathrm{C}$, 1635, 1608; $\mathrm{C}=\mathrm{N} 1502 ; \quad \mathrm{C}-\mathrm{O} \quad 1228 ; \quad{ }^{1} \mathrm{H} \quad \mathrm{NMR}$ $\left(400 \mathrm{MHz}, \mathrm{MeOH}-\mathrm{d}_{4}\right) \quad \delta 7.62(\mathrm{dd}, J=8.8 \mathrm{~Hz}, 4.4 \mathrm{~Hz} ; 2 \mathrm{H})$, $7.51(\mathrm{~m}, 2 \mathrm{H}), 7.46(\mathrm{~d}, J=13.6 \mathrm{~Hz} ; 2 \mathrm{H}), 7.37(\mathrm{~m}, 2 \mathrm{H}), 7.26(\mathrm{~m}$, 2H) $7.08(\mathrm{~m}, 2 \mathrm{H}), 6.98(\mathrm{dd}, J=6.4 \mathrm{~Hz}, 2.2 \mathrm{~Hz} ; 2 \mathrm{H}), 6.72$ $(\mathrm{dd}, J=6.4 \mathrm{~Hz}, 2.2 \mathrm{~Hz} ; 2 \mathrm{H}), \quad 6.64 \quad(\mathrm{~m}, \quad 2 \mathrm{H}), \quad 6.51$ $(\mathrm{d}, J=16.4 \mathrm{~Hz} ; 2 \mathrm{H}), 6.24(\mathrm{~d}, J=16.0 \mathrm{~Hz} ; 2 \mathrm{H}), 3.95(\mathrm{~d}, 4 \mathrm{H})$, $3.80(\mathrm{~s} ; 12 \mathrm{H}), 2.83(\mathrm{~s} ; 4 \mathrm{H}), 1.81(\mathrm{~m} ; 4 \mathrm{H}), 1.17(\mathrm{~m} ; 4 \mathrm{H}) .{ }^{13} \mathrm{C}$ NMR (400 MHz, MeOH-d $\left.{ }_{4}\right) \quad \delta$ 184.7, 150.5, 149.4, 142.2, $141.5,131.2,128.5,126.7,124.1,123.6,122.2$, 116.6, 111.7, $72.9,56.4,29.9,23.6,19.5$.

\subsection{Preparation of AgNPs supported on Cur-1,4}

In a typical preparation of supported AgNPs, $20 \mathrm{mM}$ $(0.1648 \mathrm{~g})$ of Cur-1,4 was suspended in $100 \mathrm{~mL}$ of $0.5 \times$ $10^{-3} \mathrm{M} \mathrm{AgNO}_{3}$ aqueous solution. Immediately after addition of the diimine, the initial colourless aqueous solution of $\mathrm{AgNO}_{3}$ turns into a light yellow coloured colloidal solution. The colour deepens on time towards dark yellow, yellowgreen, and finally to a dark olive green solution. The mixture was left undisturbed for $3 \mathrm{~h}$ at room temperature (Figure 1). The completion of the reaction process is marked by the formation of AgNPs which settles at the bottom of the glass vial. Then the mixture was centrifuged, washed with ethanol/distilled water and dried at room temperature to obtain the stabilized AgNPs.

\subsection{Catalytic study of stabilized AgNPs in the reduction of nitroaromatics}

The reduction of nitroaromatics was studied by using UVvisible spectrophotometry following a reported method with minor modification. ${ }^{42}$ In a typical reaction, $1 \mathrm{~mL}$ of an aqueous solution of 4-nitrophenol (1 mM) was mixed with $25 \mathrm{~mL}$ of distilled water containing $0.1 \mathrm{mg}$ of the AgNPs. The suspension was then stirred at room temperature in the inert atmosphere of $\mathrm{N}_{2}$ for $30 \mathrm{~min}$. The reduction process was started by the addition of $4 \mathrm{~mL}$ of a freshly prepared aqueous solution of $\mathrm{NaBH}_{4}(0.33 \mathrm{M})$. The degradation process was monitored by UV-Visible spectrophotometer. An aliquot of $1.5 \mathrm{~mL}$ was withdrawn in a cuvette of optical

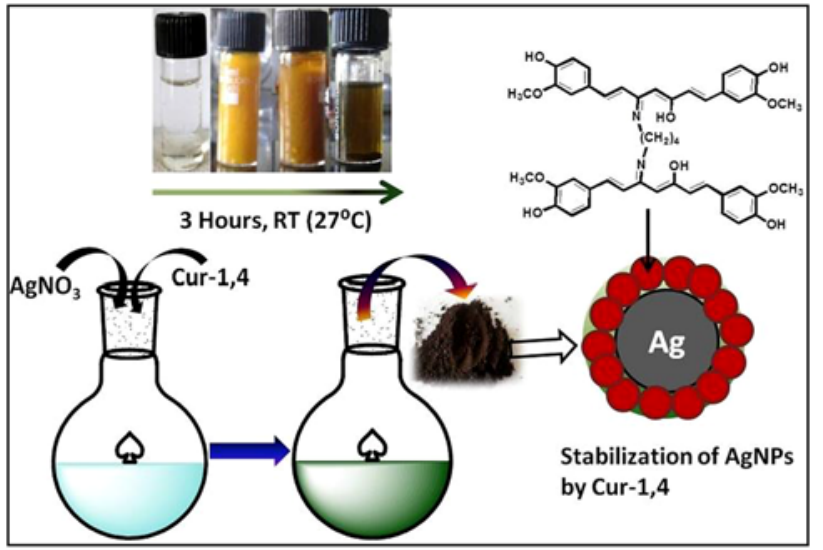

Figure 1. Preparation of AgNPs using Cur-1,4 as support/ stabilizer/reductant.

path length $1 \mathrm{~cm}$ at a regular interval and the absorbance was measured in the range of $200-800 \mathrm{~nm}$. The process of reduction was monitored by measuring the change in absorbance at $400 \mathrm{~nm}$. The catalyst was separated by centrifugation after completion of the reaction. The product was extracted with diethyl ether/dichloromethane and concentrated by a rotary evaporator for GC-MS analysis.

\subsection{Study of antibacterial activity in vitro}

The in vitro antibacterial activities of Curcumin, Cur-1,4 and AgNPs stabilized by Cur-1,4 were evaluated at a single dose $(50 \mu \mathrm{g} / \mathrm{disc})$ against two Gram-positive bacteria viz., Bacillus subtilis and Staphylococcus aureus, and two Gramnegative bacteria viz., Escherichia coli and Pseudomonas aeruginosa by agar diffusion method using Mueller-Hinton agar media. The activity of the compound was assessed by measuring the diameter of zone of inhibition of bacterial growth in $\mathrm{mm}$, and comparing with a standard antibiotic, ciprofloxacin. The stock cultures of bacteria used were basically bacterial cultures maintained at $4{ }^{\circ} \mathrm{C}$ in their appropriate agar slants throughout the study. The sub-cultured test organisms were prepared using nutrient agar medium. Bacterial strains were inoculated in the respective test tubes containing a sterilized medium. Followed by incubation at $37 \pm 1^{\circ} \mathrm{C}$ for $24 \mathrm{~h}$, they were stored in a refrigerator $\left(2-8{ }^{\circ} \mathrm{C}\right)$ and maintained as stock cultures. Bacterial inoculums for each strain were prepared by transferring a $0.005 \mathrm{~mL}$ of stock culture into a clean and sterilized tube containing 4-5 mL nutrient broth medium. The broth cultures were then incubated at $37 \pm 1{ }^{\circ} \mathrm{C}$ for 18 h. A sterilized disk (6.0 mm diameter) Whatman filter paper No. 2 impregnated with DMSO was used as the negative control. Under aseptic condition, empty sterilized disks were impregnated with the test drug solutions of $10 \mu \mathrm{L}(50 \mu \mathrm{g} / \mathrm{disk})$. After incubation of the plates at $37 \pm$ $1{ }^{\circ} \mathrm{C}$ for $18 \mathrm{~h}$, the diameter of the zones of complete inhibition surrounding each of the wells was measured in millimeter scale. Triplicate studies were performed and results 
are presented as mean values \pm standard deviation (SD). The detailed procedure of antibacterial study is included in the supplementary information.

\section{Results and Discussion}

Schiff base of curcumin with 1,4-diaminobutane (Cur1,4) was synthesized via a Mannich reaction as discussed in Scheme 1. Curcumin exists in its $\beta$-diketo form in acidic $\mathrm{pH} .{ }^{11}$ One of the keto groups of each curcumin unit is covalently bonded with the two amino groups of the 1,4-diamine moiety. The resultant product contains four phenolic functionalities and two imine functionalities. The compound obtained is solid and crystalline. The color of the compound is brick red. Elemental analyses were done and the $\%$ of $\mathrm{C}, \mathrm{H}$, $\mathrm{N}$ and $\mathrm{O}$ were found to tally approximately with that of calculated values.

\subsection{Characterization of Cur-1,4}

To confirm the formation of Cur-1,4, FTIR and ${ }^{1} \mathrm{H}$ NMR and ${ }^{13} \mathrm{C}$ NMR spectra were recorded. Formation of Cur1,4 is confirmed by a stretching vibration $v_{\mathrm{C}=\mathrm{N}}$ at $1502 \mathrm{~cm}^{-1}$ and disappearance of $v_{\mathrm{C}-\mathrm{CO}-\mathrm{C}}$ vibrational peak at $1110 \mathrm{~cm}^{-1}$ (Figure S2, Supplementary Information). The ${ }^{1} \mathrm{H}$ NMR spectrum of Cur-1,4 is shown in Figure S3, Supplementary Information. The appearance of a singlet at $\delta 3.80 \mathrm{ppm}$ showed the presence of methoxy groups in the final product. The peaks in the range of $\delta 6.24-7.62 \mathrm{ppm}$ are accounted for aromatic protons and the protons attached to the conjugated double bonds. The ${ }^{13}$ C NMR spectra of Cur-1,4 is shown in Figure S4 (Supplementary Information). The peak at $\delta 184.7 \mathrm{ppm}$ is due to the other carbonyl $\mathrm{C}=\mathrm{O}$ carbon (shown in Figure $\mathrm{S} 4$ in enol form). Imine $(\mathrm{C}=\mathrm{N})$ functionalized $\mathrm{C}$ atom shows a peak at the chemical shift value of $\delta 150.5 \mathrm{ppm}$. An intense peak originating from the $\mathrm{C}$ - atoms of the four methoxy groups appear at chemical shift value of $\delta 72.9 \mathrm{ppm}$.

\subsection{Cur-1,4 as stabilizer in AgNPs synthesis}

The role of this Schiff base (Cur-1,4) as support/stabilizer/reductant in preparation of AgNPs was studied. A typical procedure for preparing stabilized AgNPs is illustrated in Figure 1. Cur-1,4 when added to the aqueous solution of $\mathrm{AgNO}_{3}$, acts as an efficient ligand to chelate $\mathrm{Ag}^{+}$ions through its four phenolic hydroxyl $(\mathrm{OH})$ groups and/or quinones formed due to oxidation of phenolic hydroxyls. ${ }^{43}$ Due to the high redox potential of $\mathrm{Ag}^{+}$, the chelated $\mathrm{Ag}^{+}$ions could be reduced into $\mathrm{Ag}^{0}$ atoms at room temperature $\left(27^{\circ} \mathrm{C}\right)$ in situ without requiring any other chemical reducing agents.

The formation of AgNPs can be visually witnessed by the change in colour of the solution of Cur-1,4 from brick red to dark green. It is known that smaller sized AgNPs have more negative potentials compared to the bigger nanoparticles (forms through the aggregation of AgNPs in absence of a stabilizing/capping group) due to the high surface to volume ratio. Cur-1,4 shield the AgNPs to agglomerate leading to the formation of smaller sized AgNPs stabilized by the unreacted phenolic hydroxyl as well as quinone groups of Cur-1,4 (through electron donor/acceptor relations). ${ }^{44}$ In this way, Cur-1,4 supports AgNPs in maintaining the higher surface energy required for showing its higher catalytic activity (due to a higher potential difference between AgNPs and reacting substrate) and bactericidal properties. A comparative study on water-based synthetic protocols on the use of reducing and capping agents, solvent, reaction speed, temperature requirement and equipment is listed in Table S1 (Supplementary Information). Some of the methodologies carried out in an aqueous medium either require a high temperature for synthesis, long reaction time or external reducing agents. Cur-1,4 synthesized in the present work aids in preparation of AgNPs in room temperature and in water and itself acts as both reducing and stabilizing agent. Moreover, the reaction is performed without employing any equipment or

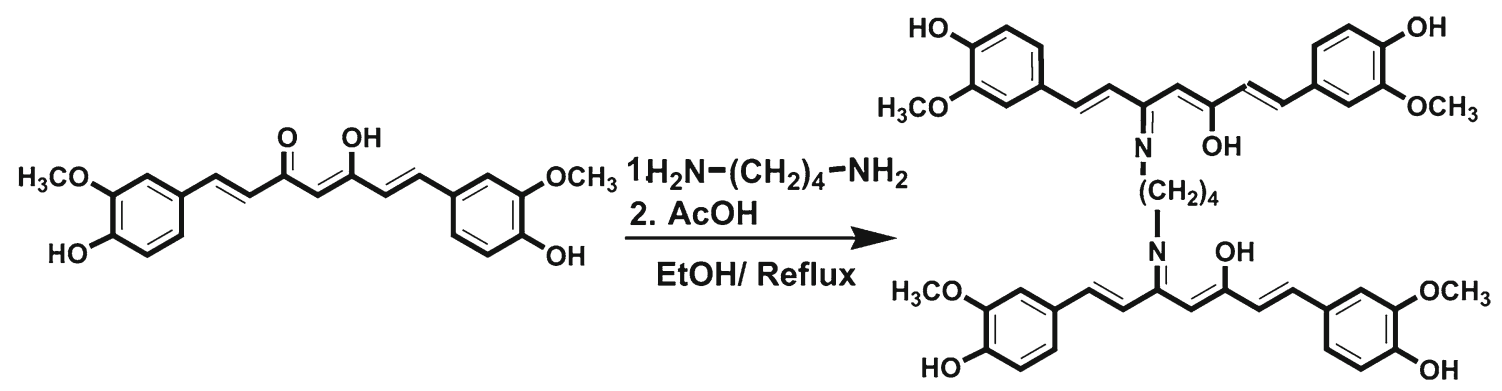

Scheme 1. Synthesis of Schiff base of curcumin with 1,4-diaminobutane (Cur-1,4). 
reaction setup. Hence, it can be concluded that our synthetic methodology fulfils the conditions of green chemistry principles in terms of - energy consumption, reaction speed, waste production, solvent medium and simple and safe operating conditions.

\subsection{Characterisation of silver nanoparticles (AgNPs)}

To confirm the formation of AgNPs, these were collected after washing by solvents (ethanol/water), centrifuged and characterized by various analytical techniques.

3.3a Fourier transform infrared (FTIR) spectroscopy: The FTIR spectra of stabilized AgNPs in Figure S2 (Supplementary Information) indicates the formation of Cur-1,4 stabilized AgNPs. The peak at $3384 \mathrm{~cm}^{-1}$ is due to the stretching vibration of the phenolic-OH bond, appears broader and is shifted to longer wavenumber compared to its native the stretching vibration in Cur-1,4. Furthermore, characteristic stretching vibrations of $\mathrm{C}=\mathrm{N}, \mathrm{C}-\mathrm{O}$, $\mathrm{C}=\mathrm{C}$ bonds are observed in the FT-IR spectra of AgNPs, indicating the presence of Cur-1,4 as a stabilizer in AgNPs. An overlaid FTIR spectra of curcumin, Cur1,4 and stabilized AgNPs is shown in Figure 2.

3.3b X-ray Diffraction (XRD) and energy-dispersive $X$-ray spectroscopy (EDS): The morphology and crystallized structure of AgNPs were characterized by taking images of XRD and EDS patterns (Figure 3a and $3 \mathrm{~b})$. All the major peaks in XRD pattern at $2 \theta=38.18^{\circ}, 44.29^{\circ}, 64.43^{\circ}, 77.48^{\circ}$ and $81.54^{\circ}$ corresponding to the crystal planes (111), (200), (220), (311) and (222) of silver are observed and are in a good agreement with Joint Committee on Powder Diffraction Standards (JCPDS) silver file No. 04-0783. ${ }^{40,45}$ The crystalline nature of synthesized AgNPs is reflected from the high intensity of peaks as observed from the XRD image and the relative high intense peak is for (111) Bragg reflection. The XRD pattern confirms the resultant particles as crystallized face centered cubic (FCC) AgNPs. The crystallite size of AgNPs estimated from Debye-Scherrer's formula is $30.02 \mathrm{~nm}$. Elemental mapping by EDS pattern shows the presence of $\mathrm{Ag}$ metal along with $\mathrm{C}, \mathrm{O}$ and $\mathrm{N}$ elements, confirming the presence of Cur-1,4 along with Ag in the synthesized stabilized AgNPs. The high intense peak of Ag in EDS pattern is due to the abundant presence of Ag compared to other elements in the nanoparticles confirming the nanoparticles to be of silver.

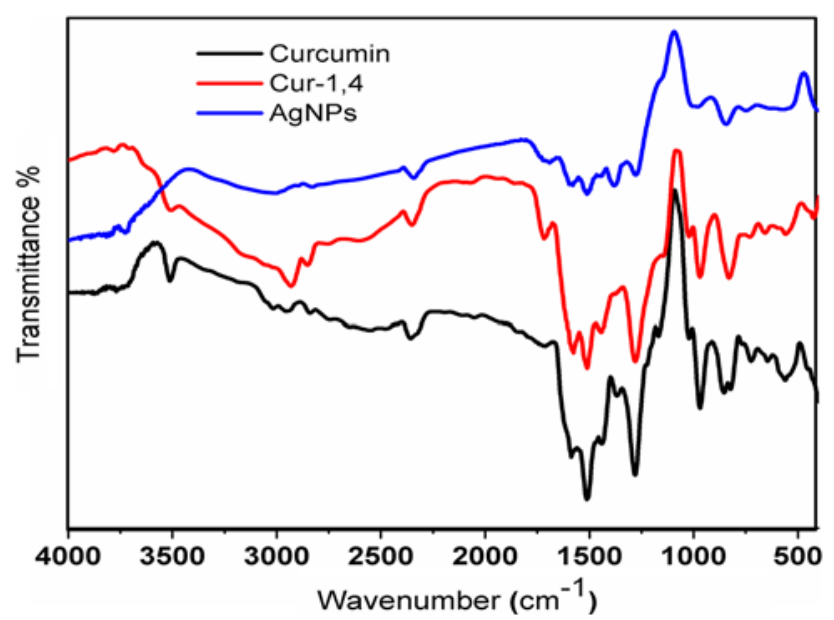

Figure 2. Overlaid FTIR spectra of Curcumin, Cur-1,4 and AgNPs stabilized by Cur-1,4.
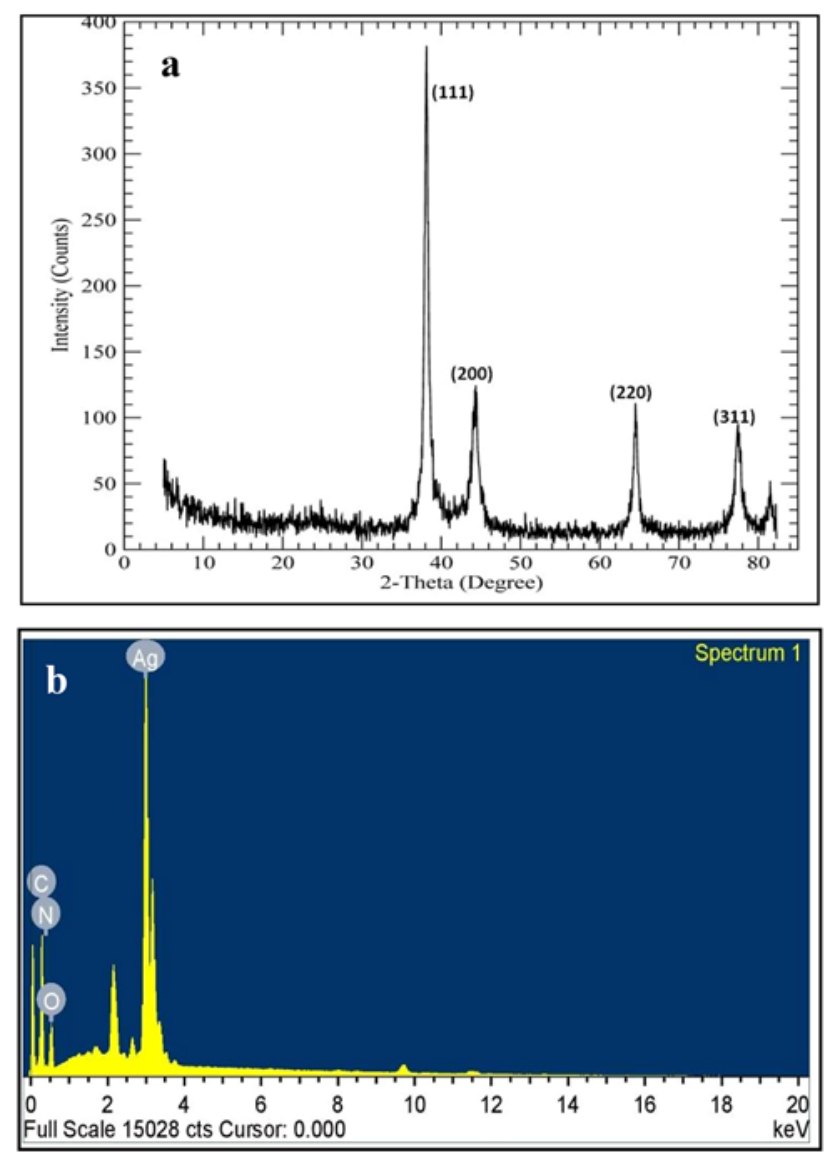

Figure 3. (a) XRD pattern; (b) EDS spectra of the synthesised AgNPs stabilized by Cur-1,4.

3.3c Scanning electron microscopy (SEM) and transmission electron microscopy (TEM): The surface morphology, particle size and size distribution of AgNPs were further determined by analysis of SEM and TEM images depicted in Figures 4 and 5. The obtained TEM and SEM images show that the sizes of 


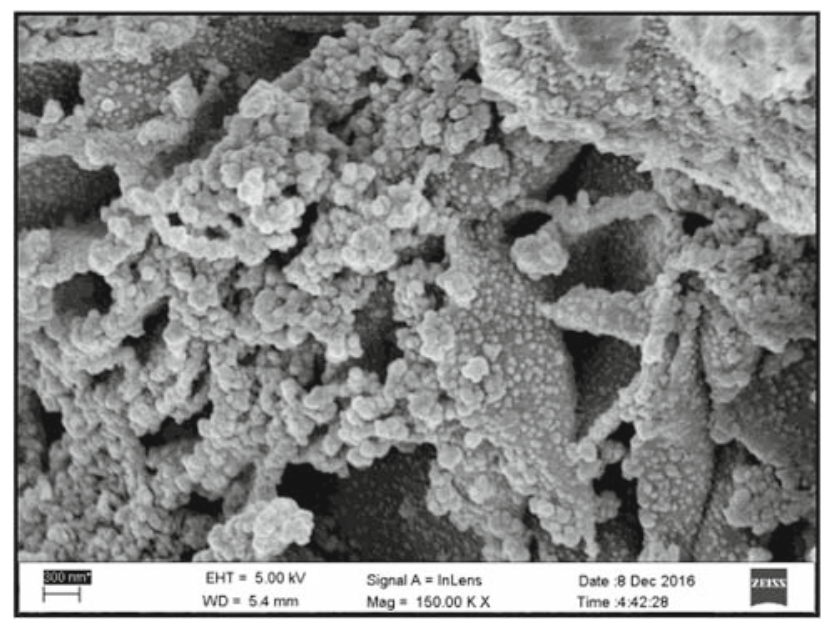

Figure 4. SEM image of AgNPs stabilized by Cur-1,4.

spherical shaped AgNPs are in the nanometer range and are dispersed in a thin organic layer of Cur-1,4, responsible for enhancing the stability of AgNPs.
Figure 6a shows the selected area of electron diffraction pattern of a spherical silver nanoparticle. The clear bright circular rings in the typical SAED pattern corresponds to the (111), (200), (220) and (311) planes of the fcc crystallized spherical AgNPs confirming the crystalline nature of AgNPs. The particle size distribution pattern in Figure $6 \mathrm{~b}$ is determined by randomly selecting 100 particles from the TEM image and AgNPs in the size ranges of 15-55 $\mathrm{nm}$ are observed. The particle size distribution diagram is narrow and most of the AgNPs fall in the average size of $25-30 \mathrm{~nm}$.

\subsection{Catalytic activity of AgNPs}

Degradation of nitroaromatic compounds is important from an environmental point of view. Since, nitro compounds, which otherwise find wide applications in agriculture field as pesticides, herbicides and textile industries, persist in the environment and start to

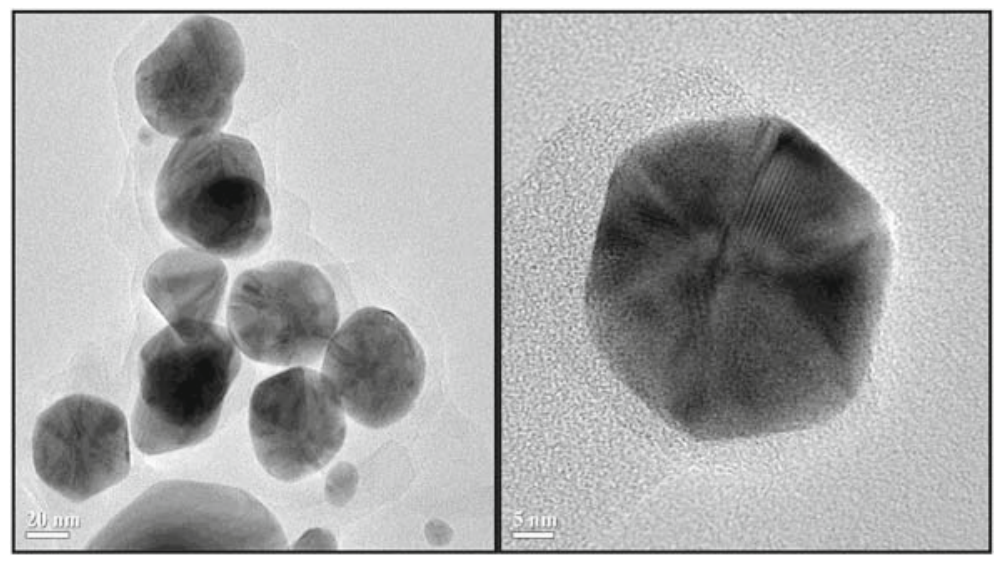

Figure 5. TEM images of AgNPs stabilized by Cur-1,4 at different magnification scales.

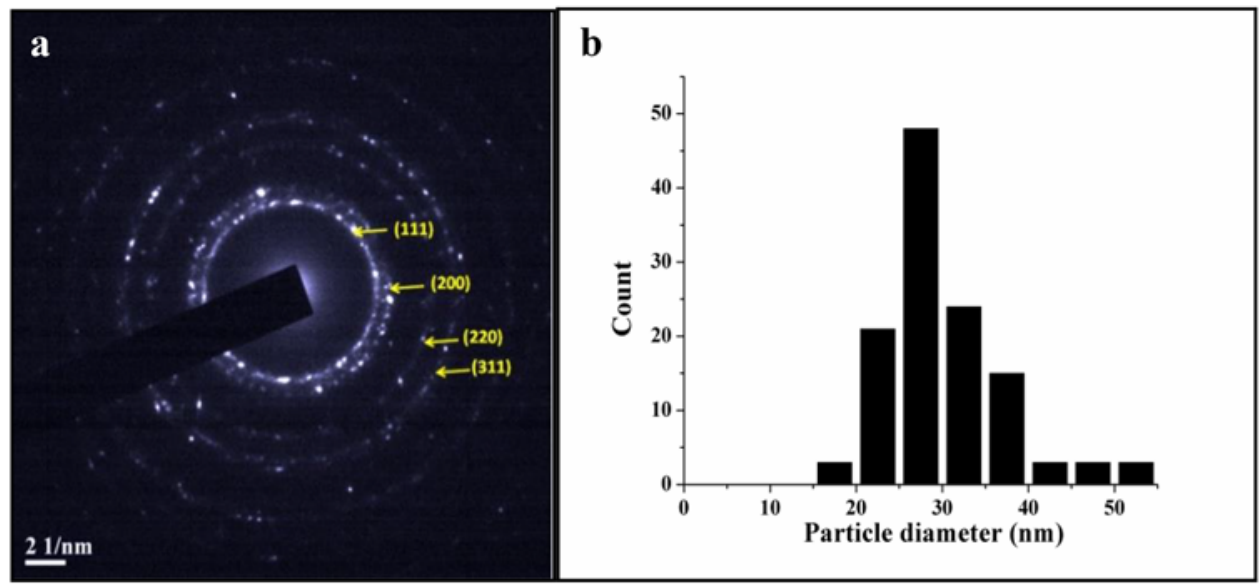

Figure 6. (a) Selected area diffraction pattern of AgNPs stabilized by Cur-1,4; (b) Particle size distribution diagram of AgNPs. 


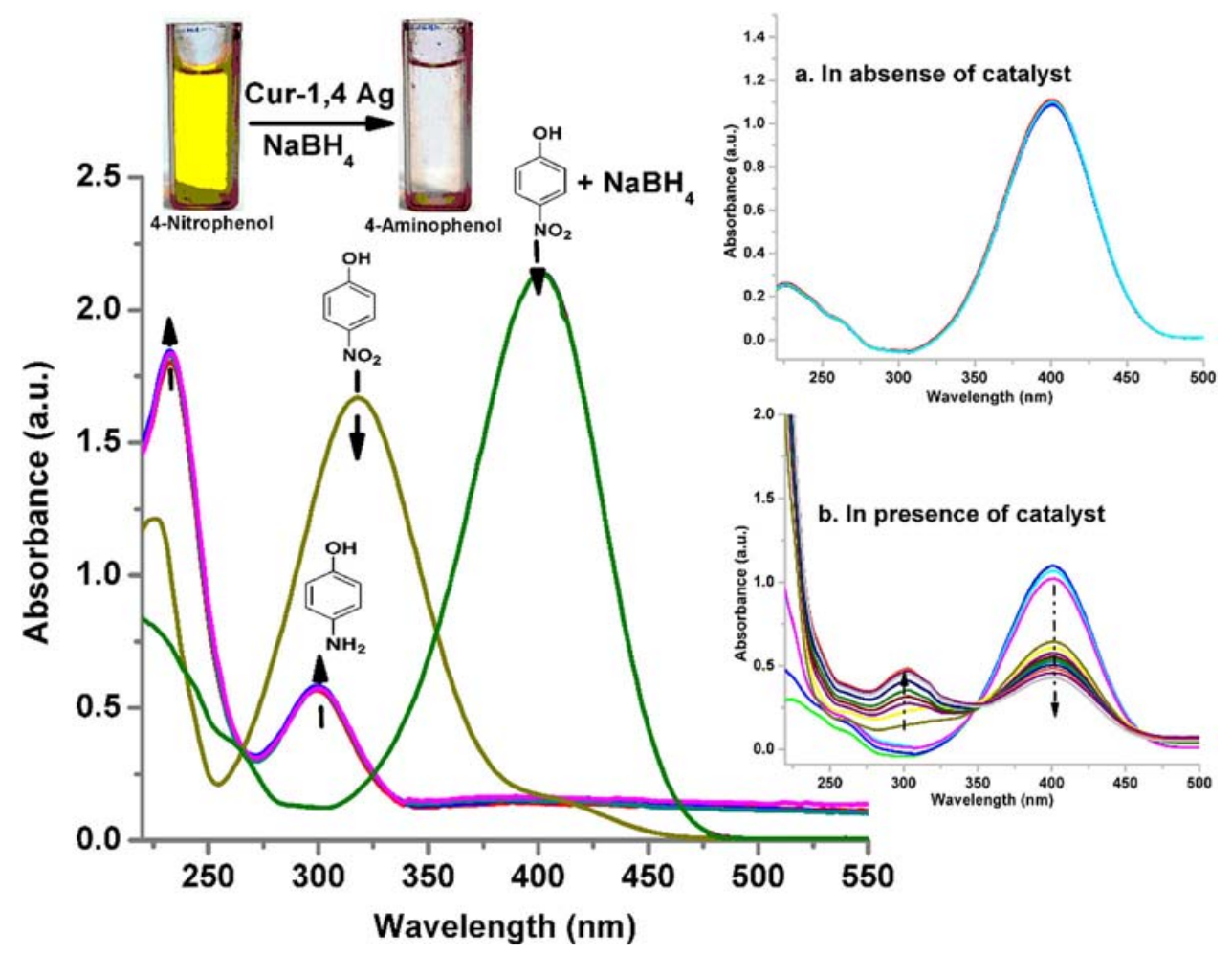

Figure 7. Monitoring of the reduction of 4-Nitrophenol (1 mM) to 4-Aminophenol by UV-vis absorption spectra (of optical path length $1 \mathrm{~cm}$ ) after addition of $0.33 \mathrm{M} \mathrm{NaBH}_{4}$ and $(0.1 \mathrm{mg}) \mathrm{AgNPs}$ stabilised by Cur-1,4 as catalyst; inset (a) in absence of Cur-1,4 AgNPs and (b) in presence of Cur-1,4 AgNPs.

contaminate the environment if left untreated. Therefore the conversion of nitro substrates to safe and environmentally benign products is highly important. $^{46-48}$ The reduction of 4-nitrophenol to 4aminophenol is mostly studied as a model reaction to evaluate the catalytic activity of noble metal nanoparticles and is commonly reported in the literature. Recently, many safe and greener methods are developed for the reduction of nitroaromatics. ${ }^{46}$ In our work we have screened the degradation of some nitroaromatics in addition to 4-nitrophenol to their corresponding amino products by Cur-1,4 stabilized AgNPs as a heterogeneous catalyst at room temperature and in an aqueous medium. The catalytic characteristic of prepared stabilized AgNPs was studied by monitoring the reduction reaction of $1 \mathrm{mM}$ aqueous solution of the nitroaromatic compound by sodium borohydride $\left(\mathrm{NaBH}_{4}\right)$ by UV-visible spectrophotometer with optical path length $1 \mathrm{~cm}$. Metal nanoparticles act as an electronic relay system from donor $\mathrm{BH}_{4}{ }^{-}$to acceptor nitro groups and can effectively catalyze the reduction of nitro compounds. As shown in Figure 7 the absorption peak of 4-nitrophenol at $317 \mathrm{~nm}$ undergoes a bathochromic shift to 400 nm immediately upon addition of $\mathrm{NaBH}_{4}$ solution, with a colour change of light yellow to yellow-green due to the formation of 4-nitrophenolate ion in alkaline condition. The absorption peak at $400 \mathrm{~nm}$ remained unaltered for a couple of days in absence of catalyst, whereas a catalytic amount of AgNPs, when added and stirred into the solution, caused decolourization of the yellow-green 4-nitrophenolate solution. The absorption peak height at $400 \mathrm{~nm}$ decreases and two new peaks at $235 \mathrm{~nm}$ and $295 \mathrm{~nm}$ are observed due to the formation of 4- aminophenol. Thus, the stabilized AgNPs act as a catalyst in the reduction of 4-nitrophenol. The AgNPs transfer electrons between nucleophilic $\mathrm{BH}_{4}^{-}$and electrophilic 4-nitrophenolate.

Apart from the reduction of 4-nitrophenol, some other nitroaromatic substrates were screened to examine the scope and limitation of Cur-1,4 stabilized AgNPs as catalysts. The reactions were monitored by UV-Visible spectrophotometer (Figure S5, Supplementary Information) and the products were analysed by GC-MS technique (Figure S7, Supplementary Information). All the reduction reactions were completed effectively, giving good yields of the corresponding amine products as indicated in Table 1. The AgNPs acted as an efficient catalyst with high catalytic activity in the process of reduction of nitroaromatic substrates irrespective of the presence of electron-donating or electron-withdrawing substituents. 
Table 1. Reduction of nitroaromatics by Cur-1,4 AgNPs in water and at r.t.

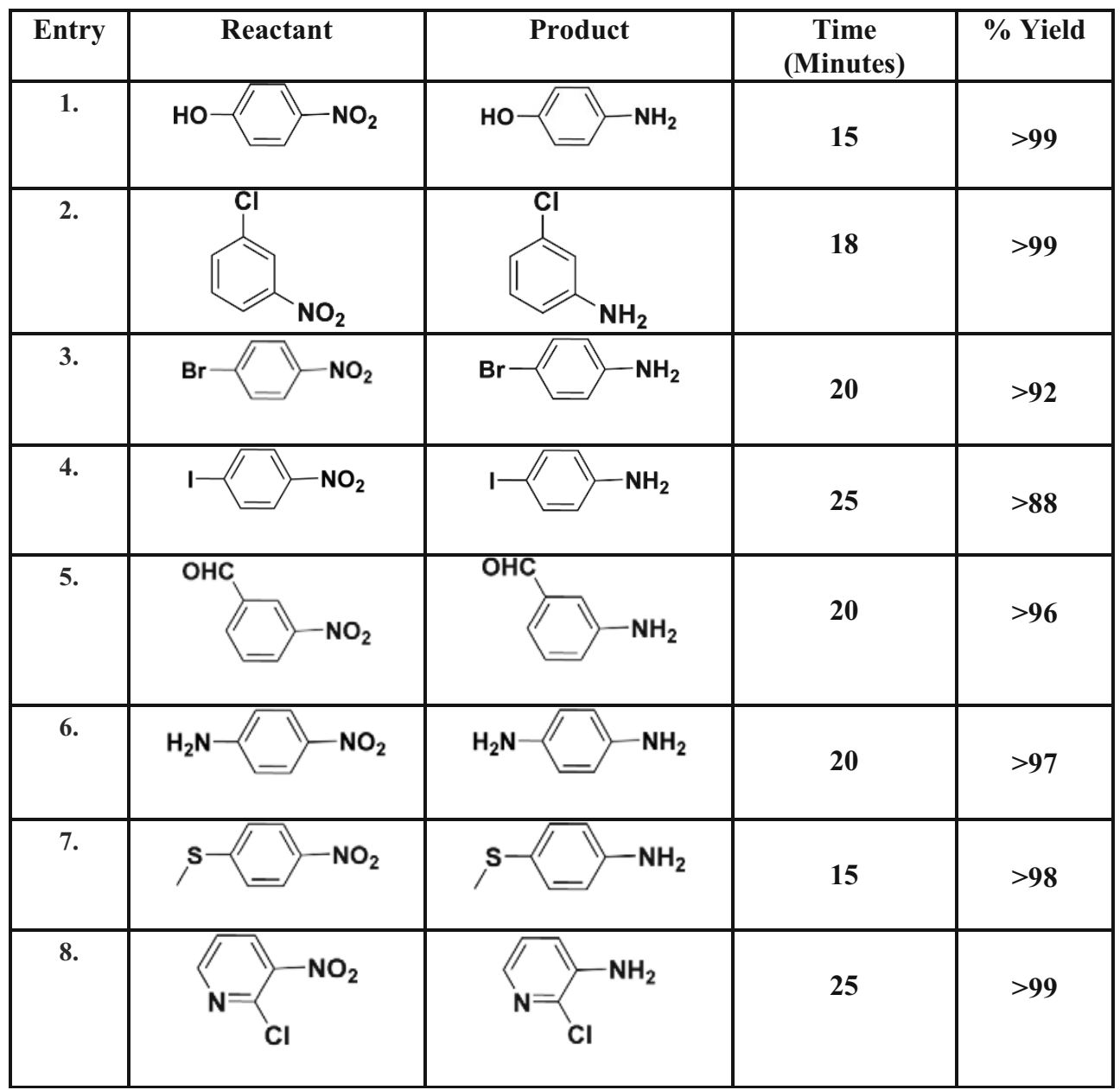

Most catalytic reactions depend on the surface structure and size of the catalysts. The plane of AgNPs containing high atom density of electrons is highly reactive. The spherical shaped AgNPs predominantly expose their highly reactive (111) face for the adsorption of the substrate (4-nitrophenol) and desorption of the product (4-aminophenol) from their surface. ${ }^{49}$ Considering the requirement of an appropriate surface area for adsorption and desorption processes it was reported that nanomaterials of metals of size $<10 \mathrm{~nm}$ have lower catalytic activity in the reduction of 4-nitrophenol to 4-aminophenol.

The stabilized AgNPs synthesized in this work are mostly spherical in shape and most of the particles are about $30 \mathrm{~nm}$ in size, as confirmed by XRD and TEM analysis. AgNPs in the size range of $25-30 \mathrm{~nm}$ can provide the desired surface area required for the adsorption of 4-nitrophenol and therefore can show effective catalytic activity.

\subsection{Catalyst leaching and its reusability}

Sustainability is the salient feature of a heterogeneous catalyst from the environment and economic concern. $^{45,49}$ Therefore, the recyclability of AgNPs as heterogeneous catalyst was investigated for the abovediscussed reduction reaction of nitroaromatics to form amino compounds. The heterogeneous nature and the leaching of $\mathrm{Ag}$ metal during the reaction of 4-nitrophenol to 4-aminophenol was investigated via a metal leaching test. After completion of the reaction, the catalyst was filtered off. Inductively coupled plasma atomic emission spectroscopy (ICP- AES) has been employed to study the filtrate collected after the $5^{\text {th }}$ cycle. A negligible amount of $\mathrm{Ag}$ metal in the filtrate (0.062 ppm) indicated insufficient leaching of $\mathrm{Ag}$ metal and thus the stabilized AgNPs are heterogeneous in nature. It was observed that the catalyst can be reused up to $5^{\text {th }}$ catalytic cycle without significant loss 
in activity (Figure S7, Supplementary Information). The heterogeneity of stabilized AgNPs makes their easy retrieval from the reaction mixture by centrifugation, which can be thoroughly washed, dried and used for the subsequent reaction cycles. The slight decrease in product yield and the time required for the product formation is due to either physical loss of stabilized AgNPs during a separation or its degradation. The catalyst is green, stable and inexpensive.

\subsection{Study on the antibacterial activity of AgNPs}

Antibacterial activities of curcumin, Cur-1,4 and stabilized AgNPs were tested against two Gram-positive (S. aureus and B. subtilis) and two Gram-negative (E. coli and $P$. aeruginosa) bacterial strains and the results are presented in Table S2 (Supplementary Information). The observed antibacterial activities of Cur-1,4 and stabilized AgNPs against the four bacterial strains i.e., S. aureus, B. subtilis, E. coli and $P$. aeruginosa, shown in Figure 8 (also in Figure S8, Supplementary Information) indicate that both of them exhibit a broad-spectrum inhibitory effect against all microorganisms. The per cent inhibition of different bacteria at $50 \mu \mathrm{g}$ (per disk) concentration solutions (DMSO) of stabilized AgNPs and Cur-1,4 followed the order: E. coli $>P$. aeruginosa $>S$. aureus $>B$. subtilis while for curcumin $(50 \mu \mathrm{g} / \mathrm{mL}$; DMSO) the order is: $S$. aureus $>B$. subtilis $>P$. aeruginosa $>$ E. coli., the results of antibacterial study indicate that (i) both Cur-1,4 and stabilized AgNPs show antibacterial properties and (ii) the selected strains of Gramnegative bacteria (E. coli and $P$. aeruginosa) have a

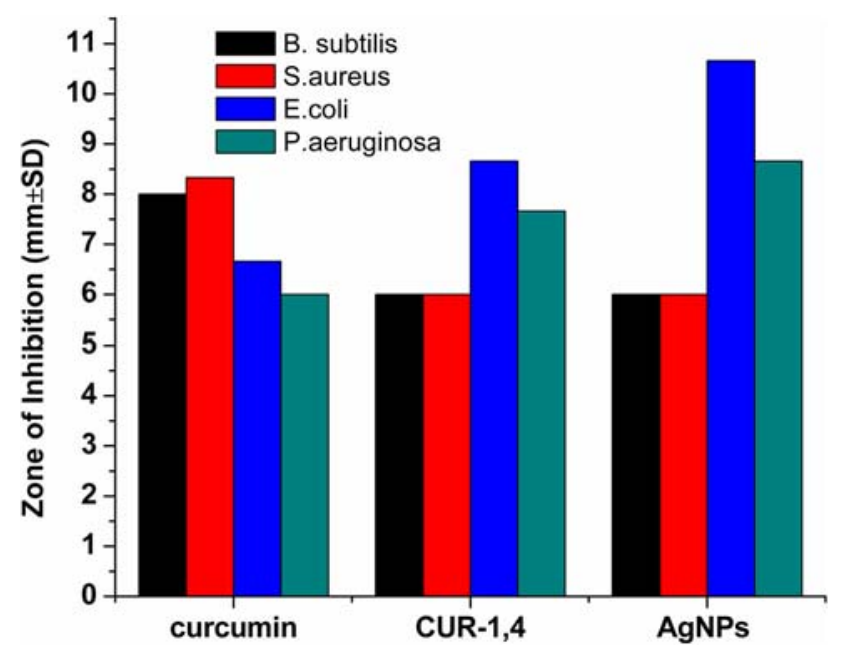

Figure 8. Zone of inhibition ( $\mathrm{mm} \pm \mathrm{SD}$ ) of B. subtilis, $S$. aureus, E. coli and P. aeruginosa by Curcumin, Cur-1,4 and stabilized AgNPs. higher sensitivity towards the Cur-1,4 and stabilized AgNPs in comparison to Gram-positive bacteria ( $S$. aureus and B. subtilis).

The antimicrobial activity of AgNPs has shape and size dependency, smaller the particles higher is their antimicrobial efficacy. Also, spherical shaped AgNPs can effectively contact the bacterial cells through their reactive (111) face. When the AgNPs are in a closer influence with the bacterial cell, oxygen generates oxidized $\mathrm{Ag}$ and silver ions $\left(\mathrm{Ag}^{+}\right)$which penetrates inside the bacterial cell and interact with the sulphur and phosphorus-containing biomolecules as DNA and proteins resulting in inhibition of DNA replication. The thiol group (-SH) of enzymes such as NADH dehydrogenases are blocked causing breakdown of the respiratory chain. Moreover, the free radicals generated from the surface of AgNPs induce oxidative stress in the cell membrane. All these factors synergistically lead to the death of bacterial cells. ${ }^{50}$

The differences in the structure and constituents of bacterial cell membrane decide the ease of permeability in the bacterial cell. It was reported that an outer liposaccharide membrane is present over a thin peptidoglycan layer in Gram-negative bacteria. But in Gram-positive bacteria only an outer thick peptidoglycan layer consisting of liposaccharide chains crosslinked by short peptides is present that makes them rigid and less permeable. ${ }^{51,52}$

\section{Conclusions}

In summary, a novel and green protocol for preparing stabilized AgNPs is developed in presence of a newly synthesised Schiff base of curcumin with 1,4-diaminobutane (Cur-1,4) as a stabilizing and self reducing agent with improvement in aqueous solubility compared to curcumin. The solubility of Cur- 1,4 in water, as well as its role as self-reducing and stabilizing agent, promotes the synthesis of AgNPs in a cost-effective, simple and greener process. TEM and XRD patterns demonstrated that the spherical AgNPs are crystallized in a face-centred (fcc) structure and a size of approximately 25-30 $\mathrm{nm}$. The stabilized AgNPs are easy to prepare in environmentally benign conditions. Moreover, these can be recovered and reused as a catalyst in reducing nitroaromatics in greener conditions. It is important from an economic and environmental perspective. The antibacterial test showed that both stabilizing agent Cur-1,4 and AgNPs have strong antibacterial activity against test bacterial strains, which is unique and this property can find importance in medicinal fields in future. 


\section{Supplementary Information (SI)}

The preparation of Cur-1,4 and characterization of stabilized Ag nanoparticles is available in supplementary information at www.ias.ac.in/chemsci.

\section{Acknowledgements}

The authors are grateful to the Department of Science and Technology, Govt. of India, for financial assistance under DST-FIST programme and UGC, New-Delhi for Special Assistance Program (SAP-DRS) to the Department of Chemistry, Dibrugarh University. The authors are also grateful to Sophisticated Analytical Instrument Facility (SAIF), NEHU, Shillong, IASST, Guwahati, CSIR NEIST, Jorhat and SAIF, IIT Bombay for spectral analysis and Department of Pharmaceutical sciences, Dibrugarh University for in vitro antibacterial studies. NG is thankful to Dibrugarh University for Dibrugarh University Research Fellowship.

\section{References}

1. Jayaprakasha G K, Rao L J and Sakariah K K 2006 Antioxidant activities of curcumin, demethoxycurcumin and bisdemethoxycurcumin Food Chem. 98720

2. Anderson A M, Mitchell M S and Mohan R S 2000 Isolation of curcumin from turmeric J. Chem. Edu. 77 359

3. Chan M M Y 1995 Inhibition of tumor necrosis factor by curcumin, a phytochemical Biochem. Pharmacol. 49 1551

4. Pan K, Zhong Q and Baek S J 2013 Enhanced dispersibility and bioactivity of curcumin by encapsulation in casein nanocapsules J. Agric. Food Chem. 61 6036

5. Ringman J M, Frautschy S A, Cole G M, Masterman D L and Cummings J L 2005 A potential role of the curry spice curcumin in Alzheimer's disease Curr. Alzheimer Res. 2131

6. Mohanty C, Acharya S, Mohanty A K, Dilnawaz F and Sahoo S K 2010 Curcumin-encapsulated MePEG/PCL diblock copolymeric micelles: a novel controlled delivery vehicle for cancer therapy Nanomedicine $\mathbf{5}$ 433

7. Fathima N N, Devi R S, Rekha K B and Dhathathreyan A 2009 Collagen-curcumin interaction- A physicochemical study J. Chem. Sci. 121509

8. Sherin D R and Rajasekharan K N 2016 Curcuminoidderived 3,5-bis(stryl)isoxazoles-Mechanochemical synthesis and antioxidant activity J. Chem. Sci. 128 1315

9. Monroy A, Lithgow G J and Alavez S 2013 Curcumin and neurodegenerative diseases Biofactors 39122

10. Aggarwal B B, Kumar A and Bharti A C 2003 Anticancer potential of curcumin: preclinical and clinical studies Anticancer Res. 23363

11. Sun Y M, Zhang H Y, Chen D Z and Liu C B 2002 Theoretical elucidation on the antioxidant mechanism of curcumin: a DFT study Org. Lett. 42909
12. Simon A, Allais D P, Duroux J L, Basly J P, DurandFontanier S and Delage C 1998 Inhibitory effect of curcuminoids on MCF-7 cell proliferation and structure-activity relationships Cancer Lett. 129111

13. Sahu P K 2016 Design, structure activity relationship, cytotoxicity and evaluation of antioxidant activity of curcumin derivatives/analogues Eur. J. Med. Chem. 121510

14. Kundu S and Nithiyanantham U 2013 In situ formation of curcumin stabilized shape-selective Ag nanostructures in aqueous solution and their pronounced SERS activity $R S C A d v .325278$

15. Sharma R A, Gescher A J and Steward W P 2005 Curcumin: the story so far Eur. J. Cancer 411955

16. Wang D, Veena M S, Stevenson K, Tang C, Ho B, Suh J D and Wang M B 2008 Liposome-encapsulated curcumin suppresses growth of head and neck squamous cell carcinoma in vitro and in xenografts through the inhibition of nuclear factor $\kappa \mathrm{B}$ by an AKT-independent pathway Clin. Cancer Res. 146228

17. Gupta V, Aseh A, Ríos C N, Aggarwal B B and Mathur A B 2009 Fabrication and characterization of silk fibroin-derived curcumin nanoparticles for cancer therapy Int. J. Nanomed. 4115

18. Das R K, Kasoju N and Bora U 2010 Encapsulation of curcumin in alginate-chitosan-pluronic composite nanoparticles for delivery to cancer cells Nanomed. 6153

19. Maiti K, Mukherjee K, Gantait A, Saha B P and Mukherjee P K 2007 Curcumin-phospholipid complex: preparation, therapeutic evaluation and pharmacokinetic study in rats Int. J. Pharm. 330155

20. Yallapu M M, Jaggi M and Chauhan S C 2010 Poly ( $\beta$ cyclodextrin)/Curcumin Self-Assembly: A Novel Approach to Improve Curcumin Delivery and its Therapeutic Efficacy in Prostate Cancer Cells Macromol. Biosci. 101141

21. Massaro M, Amorati R, Cavallaro G, Guernelli S, Lazzara G, Milioto S and Riela S 2016 Direct chemical grafted curcumin on halloysite nanotubes as dual-responsive prodrug for pharmacological applications Colloid. Surf. B $\mathbf{1 4 0} 505$

22. Chandrasekar T, Pravin N and Raman N 2014 Biosensitive metal chelates from curcumin analogues: DNA unwinding and anti-microbial evaluation Inorg. Chem. Commun. 4345

23. Fang L, Gou S, Liu X, Cao F and Cheng L 2014 Design, synthesis and anti-Alzheimer properties of dimethylaminomethyl-substituted curcumin derivatives Bioorg. Med. Chem. Lett. 2440

24. Fang X, Fang L, Gou S and Cheng L 2013 Design and synthesis of dimethylaminomethyl-substituted curcumin derivatives/analogues: Potent antitumor and antioxidant activity, improved stability and aqueous solubility compared with curcumin Bioorg. Med. Chem. Lett. 231297

25. Zhang L, Yu J C, Yip H Y, Li Q, Kwong K W, Xu A W and Wong P K 2003 Ambient light reduction strategy to synthesize silver nanoparticles and silver-coated $\mathrm{TiO} 2$ with enhanced photocatalytic and bactericidal activities Langmuir 1910372

26. Gong C, Wu Q, Wang Y, Zhang D, Luo F, Zhao X and Qian Z 2013 A biodegradable hydrogel system 
containing curcumin encapsulated in micelles for cutaneous wound healing Biomaterials 346377

27. Panáček A, Kvitek L, Prucek R, Kolář M, Večeřová R, Pizúrová N and Zbořil R 2006 Silver colloid nanoparticles: synthesis, characterization, and their antibacterial activity J. Phys. Chem. B 11016248

28. Chou K S and Ren C Y 2000 Synthesis of nanosized silver particles by chemical reduction method Mater. Chem. Phys. 64241

29. Huang H H, Ni X P, Loy G L, Chew C H, Tan K L, Loh F C and Xu G Q 1996 Photochemical formation of silver nanoparticles in poly(N-vinylpyrrolidone) Langmuir 12909

30. Zhang Z, Zhao B and $\mathrm{Hu}$ L 1996 PVP protective mechanism of ultrafine silver powder synthesized by chemical reduction processes J. Solid State Chem. 121 105

31. Chen M, Wang L Y, Han J T, Zhang J Y, Li Z Y and Qian D J 2006 Preparation and study of polyacryamide-stabilized silver nanoparticles through a one-pot process J. Phys. Chem. B 11011224

32. Chou C W, Hsu S H, Chang H, Tseng S M and Lin H R 2006 Enhanced thermal and mechanical properties and biostability of polyurethane containing silver nanoparticles Polym. Degrad. Stab. 911017

33. Lee M H, Oh S G, Suh K D, Kim D G and Sohn D 2002 Preparation of silver nanoparticles in hexagonal phase formed by nonionic Triton X-100 surfactant Colloid. Surf. A 21049

34. Liz-Marzán L M and Lado-Touriño I 1996 Reduction and stabilization of silver nanoparticles in ethanol by nonionic surfactants Langmuir 123585

35. Raveendran P, Fu J and Wallen S L 2003 Completely "green" synthesis and stabilization of metal nanoparticles J. Am. Chem. Soc. 12513940

36. Mandal S, Selvakannan P R, Pasricha R and Sastry M 2003 Keggin ions as UV-switchable reducing agents in the synthesis of Au core- Ag shell nanoparticles J. Am. Chem. Soc. 1258440

37. Patolsky F, Lichtenstein A and Willner I 2001 Electronic transduction of DNA sensing processes on surfaces: amplification of DNA detection and analysis of single-base mismatches by tagged liposomes J. Am. Chem. Soc. 1235194

38. Pastoriza-Santos I and Liz-Marzán L M 2002 Synthesis of silver nanoprisms in DMF Nano Lett. 2903

39. El Khoury E, Abiad M, Kassaify Z G and Patra D 2015 Green synthesis of curcumin conjugated nanosilver for the applications in nucleic acid sensing and anti-bacterial activity Colloid. Surf. B 127274

40. Verma A D, Jain N, Singha S K, Quraishi M A and Sinha I 2016 Green synthesis and catalytic application of curcumin stabilized silver nanoparticles J. Chem. Sci. 1281871

41. Singh D K, Jagannathan R, Khandelwal P, Abraham P M and Poddar P 2013 In situ synthesis and surface functionalization of gold nanoparticles with curcumin and their antioxidant properties: an experimental and density functional theory investigation Nanoscale $\mathbf{5}$ 1882

42. Bhatia N K, Kishor S, Katyal N, Gogoi P, Narang P and Deep S 2016 Effect of $\mathrm{pH}$ and temperature on conformational equilibria and aggregation behaviour of curcumin in aqueous binary mixtures of ethanol $R S C$ $A d v .6103275$

43. He L, Wu H, Gao S, Liao X, He Q and Shi B 2012 Silver nanoparticles stabilized by tannin grafted collagen fiber: synthesis, characterization and antifungal activity Ann. Microbial. 62319

44. Maity D, Bain M K, Bhowmick B, Sarkar J, Saha, S, Acharya K and Chattopadhyay D 2011 In situ synthesis, characterization, and antimicrobial activity of silver nanoparticles using water soluble polymer J. Appl. Polym. Sci. 1222189

45. Xu R, Wang D, Zhang J and Li Y 2006 Shape-dependent catalytic activity of silver nanoparticles for the oxidation of styrene Chem. Asian J. 1888

46. Zhang K, Suh J M, Choi J W, Jang H W, Shokouhimehr M and Varma R S 2019 Recent advances in the nanocatalyst-assisted $\mathrm{NaBH}_{4}$ reduction of nitroaromatics in water ACS Omega 4483

47. Bhosale M A, Chenna D R and Bhanage B M 2017 Ultrasound assisted synthesis of gold nanoparticles as an efficient catalyst for reduction of various nitro compounds ChemistrySelect 21225

48. An Q, Yu M, Zhang Y, Ma W, Guo J and Wang C 2012 $\mathrm{Fe}_{3} \mathrm{O}_{4} @$ Carbon microsphere supported Ag-Au bimetallic nanocrystals with the enhanced catalytic activity and selectivity for the reduction of nitroaromatic compounds J. Phys. Chem. C 11622432

49. Kästner C and Thünemann A F 2016 Catalytic reduction of 4-nitrophenol using silver nanoparticles with adjustable activity Langmuir 327383

50. Chen S, Wu G and Zeng H 2005 Preparation of high antimicrobial activity thiourea chitosan- $\mathrm{Ag}{ }^{+}$complex Carbohyd. Polym. 6033

51. Acharya D, Singha K M, Pandey P, Mohanta B, Rajkumari J and Singha L P 2018 Shape dependent physical mutilation and lethal effects of silver nanoparticles on bacteria Sci. Rep. 8201

52. Basniwal R K, Buttar H S, Jain V K and Jain N 2011 Curcumin nanoparticles: preparation, characterization, and antimicrobial study J. Agric. Food Chem. 59 2056 